\title{
Evaluation of the Safety and Effectiveness of the Rapid Flow Expulsion Maneuver to Clear Subglottic Secretions In Vitro and In Vivo
}

In this issue of Respiratory CARE, $\mathrm{Li}$ et $\mathrm{al}^{1}$ present an approach to removing subglottic secretions. Applying the principles and physics of the normal physiologic cough maneuver, the authors demonstrated the effect of a rigorous rapid flow expulsion maneuver through an endotracheal tube in an in vitro lung model as well as in vivo.

It is well established that secretions that originate in the oropharynx and migrate to the lower respiratory tract are a primary source of respiratory infection; it is also well known that these infections present a heavy burden by way of costs and morbidity. Moreover, these infections are more likely to manifest when there is an endotracheal tube in situ, because host mechanisms are mitigated and a straighter path to the lower airways is available for secretion migration. ${ }^{2}$

The clinician has several therapeutic options to augment a patient's expulsion of tracheobronchial secretions. Suctioning through the distal end of the endotracheal tube and in the oropharynx is relatively straightforward and can be achieved effectively with a suction catheter. There are manual maneuvers and mechanistic modalities that seek to mimic, in the same way the authors have done, the normal physiologic cough maneuver. Additionally, there are techniques to mechanically remove the biofilm that resides within the artificial airway itself.

More technically challenging, however, is reaching the secretions that migrate past the glottis and rest on the top of the inflated endotracheal tube. Much effort has been put into making the removal of subglottic secretions an easier task, thereby reducing the opportunity for infection. ${ }^{3}$

One approach to this includes specially designed tracheal tubes that have a side port placed below the glottis and above the inflated cuff. This port is then attached to either continuous or intermittent wall suction, and pooling

Mr Lamb has disclosed relationships with Fisher Paykel, Sunovian Medtronic, and Bayer Pharmaceuticals. Mr Kriner has disclosed a relationship with IngMar Medical.

Correspondence: Keith D Lamb RRT-ACCS, ECMO Department, INOVA Fairfax Medical Campus, 3300 Gallows Road, Falls Church, VA 22042. E-mail: lambrrt@gmail.com.

DOI: $10.4187 /$ respcare. 05737 secretions are removed as they accumulate. When subglottic secretion removal is accomplished with these tubes (either tracheostomy or endotracheal), the incidence of ventilator-associated pneumonia is reduced, and when these

See the Original Study on Page 1007

devices are used in conjunction with semi-recumbent positioning, pneumonia is even further reduced. ${ }^{4,5}$ When multiple non-pharmacologic interventions are implemented, ventilator-associated pneumonia rates are even further decreased. ${ }^{6}$

Li et al, ${ }^{1}$ with their approach of implementing a rapid flow expulsion maneuver, potentially add to the clinician's arsenal of options to remove those subglottic secretions not removed by other modalities that expel secretions proximal to the endotracheal tube cuff. Of importance, and yet to be determined, will be the effectiveness and impact on important clinical outcomes when using the rapid flow expulsion maneuver to clear subglottic secretions. This is an important juxtaposition to the complexity and lack of independent clinician performance of the maneuver as described in the paper. ${ }^{1}$

With all of the above in mind, and when there is a clear advantage to effectively remove secretions that will potentially contaminate the lower respiratory tract, this paper by $\mathrm{Li}$ et $\mathrm{al}^{1}$ seems timely. These described procedures appear to be safe, effective, and reproducible, thereby translating nicely into the clinical setting.

Keith D Lamb RRT-ACCS INOVA Fairfax Medical Campus Falls Church, VA

Eric J Kriner RRT MedStar Washington Hospital Center Washington, D.C.

\section{REFERENCES}

1. Li J, Zong Y, Zhou Q, Dai H, Wang C. Evaluation of the safety and effectiveness of the rapid flow expulsion maneuver to clear subglottic secretions in vitro and in vivo. Respir Care 2017;62(8):1007-1013. 


\section{EDITORIALS}

2. Charles MP, Kali A, Easow JM, Joseph NM, Ravishankar, Srinivasan S, et al. Ventilator-associated pneumonia. Australas Med J 2014;7(8): 334-344.

3. Vijai MN, Ravi PR, Setlur R, Vardhan H. Efficacy of intermittent subglottic suctioning in prevention of ventilator-associated pneumonia: a preliminary study of 100 patients. Indian J Anaesth 2016;60(5):319-324.

4. Ledgerwood LG, Salgado MD, Black H, Yoneda K, Sievers A, Belafsky PC. Tracheotomy tubes with suction above the cuff reduce the rate of ventilator-associated pneumonia in intensive care unit patients. Ann Otol Rhinol Laryngol 2013;122(1):3-8.

5. Chen G, Wang J, Liu C, Xu R, Li Q, Zhou X, Gan X. Subglottic secretion drainage and semi-recumbent position for preventing ventilator associated pneumonia. Int J Clin Exp Med 2016;9(2):5193-5198.

6. Berry M, Martí JD. Clinical management of secretion retention in critically ill patients who are intubated and mechanically ventilated. Curr Respir Med Rev 2014;10(3):163-175. 